\title{
Angular Effect of Virtual Vertices Inserted to Treat The Boundary Edges on an Infinite Conducting Surface
}

\author{
Ji-Hwan Hwang $\cdot$ Soon-Koo Kweon · Yisok Oh
}

\begin{abstract}
This study presents the angular effects of virtual vertices inserted for effective treatment of the boundary edge laid on an infinite conducting surface in a half-space scattering problem. We investigated the angular effects of virtual vertices by first computing the radar cross section (RCS) of a specific scatterer; i.e., a tilted conducting plate in contact with the ground surface, by inserting the virtual vertex in half-space. Here, the electric field integral equation is used to solve this problem with various virtual vertex angles $\left(\theta_{v}\right)$ and conducting plate inclination angles $\left(\theta_{r}\right)$ ranging from $0^{\circ}$ to $180^{\circ}$. The effects of the angles $\theta_{v}$ and $\theta_{r}$ on the RCS computation are clearly shown with numerical results with and without the virtual vertices in free- and half-spaces.
\end{abstract}

Key words: Electric Field Integral Equation (EFIE), Half-Space Scattering, Image Theory, RWG Basis, Virtual Vertex.

\section{I . Introduction}

In In the field of remote sensing and earth observation utilizing microwave radar systems, the method of moments (MoM) with the Rao-Wilton-Glisson (RWG) basis function and its derivatives is widely used to compute the scattering properties of an arbitrary object [1], [2]. Recently, the use of this numerical analysis technique has been proposed in various studies to analyze the scattering property in half-space, which includes multiple scattering between scattering particles and bottom surfaces [3], [4]. Development of these numerical techniques is very useful in the remote sensing of earth terrain because the scattering process from scatterers randomly placed on a flat surface, such as a water surface or ground plane, can be represented well by a half-space scattering that applies the image theory or the impedance boundary condition [5].

In particular, due to the edges laid on the interface, the placement of a scatterer on an infinite conducting or impedance surface results in complications in computational processes such as singularity or inaccurate calculations by original sources and their images on the same surface. Image theory [6], [7] and a recent study [3] do not consider this contact problem, so some am- biguity exists for numerically solving the scattering property of a flat surface that includes various scatterers. Thus, we effectively solved this issue on the half-space scattering problem using the MoM process with RWG basis function by proposing a virtual vertex technique whereby the contact surface of the scatterer can be removed and replaced by the half-RWG basis of the boundary edge on the interface of half-space and an additionally inserted virtual vertex [4], and we clearly show its angular effect in this study.

Virtual vertices inserted for effective treatment of the problematic boundary edges in half-space scattering can be located essentially at any position of the upper halfspace, including the interface. One of the possible solutions is to place them on the interface of the half-space. In this case, a half-RWG basis vector generated by the virtual vertex has only tangential vector components, which will be canceled by their images when we apply an exact image theory to solve the half-space scattering problem, such as a conducting hemisphere laid on an infinite conducting surface [6], [7].

However, several arguments about the effect of virtual vertexes persist. For example, in the case of a scattering object that includes a scattering surface perpendicular to the ground surface, we usually encounter singular points

Manuscript received November 28, 2012 ; Revised February 18, 2013 ; Accepted February 27, 2013. (ID No. 20121128-028J)

Dept. of Electronic Information \& Communication Engineering, Hongik University, Seoul, Korea.

Corresponding Author : Yisok Oh (e-mail : yisokoh@hongik.ac.kr)

This is an Open-Access article distributed under the terms of the Creative Commons Attribution Non-Commercial License (http://creativecommons.org/licenses/ by-nc/3.0) which permits unrestricted non-commercial use, distribution, and reproduction in any medium, provided the original work is properly cited. 
when the electric field integral equation (EFIE) is used to solve the problem because the projection of orthogonal vectors becomes zero. Therefore, the virtual vertex in this case does not contribute to a computational result and even frequently creates a singularity in the computational procedure [8], [9]. In other words, when the original half-RWG basis vector and the added halfRWG basis by the virtual vertex are perpendicular, the effectiveness of the insertion of a virtual vertex becomes questionable.

We investigated the effect of the position of a virtual vertex by first introducing a conducting plate whose side (boundary edge) is attached to the ground surface, as shown in Fig. 1, with an inclination angle $\theta_{r}$; and inserting a virtual vertex at an arbitrary position with a virtual vertex angle $\theta_{v}$ and a vertex distance $d$ from the center of the boundary edge $\left(v_{\mathrm{c}}\right)$. In effect, a relatively small distance $d$ with respect to the length of boundary edge did not affect the computational result. The interaction between the virtual vertex and the boundary edge at various angles of $\theta_{v}$ and $\theta_{r}$ was then numerically simulated in the half- and free-space problems.

In the present study, we discuss the angular effect of virtual vertices. When we apply existing numerical techniques [1], [8], [9] to solve the half-space scattering problem, an optimal position for a virtual vertex will be determined to provide accurate numerical results for scattering from an arbitrary object on a conducting surface. The EFIE was used in the present study to compute these scattering problems with an open-structure, such as a conducting plate.
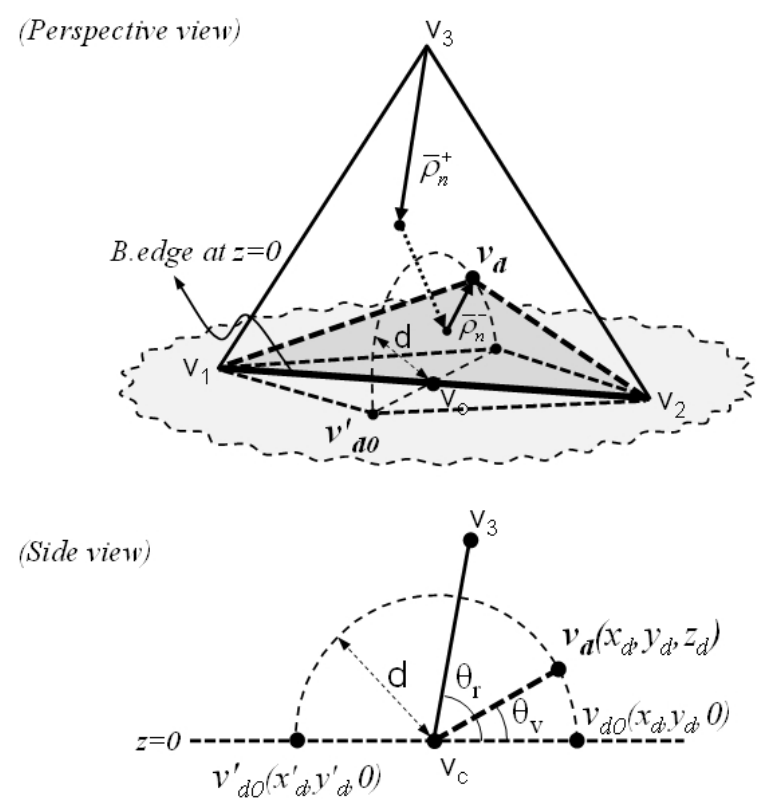

Fig. 1. Geometry of the boundary edge laid on an infinite conducting surface and arbitrarily positioned virtual vertex $\left(v_{d}\right.$ or $v_{d 0}$ on $\left.z=0\right)$.

\section{II . Virtual Vertex in Half-Space}

The geometrical parameters associated with an arbitrary virtual vertex in the upper half-space and the floating boundary edge on the interface are shown in Fig. 1. Insertion of a virtual vertex results in incorporation of a half RWG basis of the boundary edge into the MoM process to depict the vertical current components passing through it [4], [6], [7]. The angular effect of a virtual vertex in an arbitrary angle is examined by computing the scattering properties of test objects with various angles of $\theta_{v}$ and $\theta_{r}$.

\section{2-1 Modified Electric Field Integral Equation}

We computed the scattering property of a test object with various angles using the modified integral equation with the original current sources and their images. The original current sources $\bar{J}$ and their images $\bar{J}_{i}$ are expressed as shown in (1) and (2) [10].

$$
\begin{aligned}
& \bar{J}=J_{x} \hat{x}+J_{y} \hat{y}+J_{z} \hat{z} \\
& \bar{J}_{i}=-\left(J_{x} \hat{x}+J_{y} \hat{y}\right)+J_{z} \hat{z}
\end{aligned}
$$

The scattered fields $\bar{E}_{(i)}^{s}$ due to the original and image curent sources can be represented as shown in (3) [4].

$$
\begin{aligned}
& -\left.\bar{E}^{i n c}\right|_{\tan }=\left.\left(\bar{E}^{s}+\bar{E}_{i}^{s}\right)\right|_{\tan } \\
& \left.\bar{E}_{(i)}^{s}=-j \omega \mu \iint_{s^{\prime}}\left(1-\frac{1}{k^{2}} \nabla \nabla^{\prime} \cdot\right) \bar{J}_{s(i)}\left(\bar{r}_{(i)}\right)\right) \frac{e^{-j k R_{(i)}}}{4 \pi R_{(i)}} d \bar{r}^{\prime}
\end{aligned}
$$

where subscript ' $i$ ' implies image current sources to depict the reflected waves from the infinite ground plane in the half-space. We should mention that the sum of scattered fields in (3a) must be calculated separately for vertical and horizontal components with respect to the ground plane because the horizontal vector components have opposite directions in their images. The scattered fields $\bar{E}_{(i)}^{s}$ were computed using numerical integration with Gaussian quadrature over triangle [8] and analytic integration for singularity extraction from self-cells [9]. In particular, this analytic integration may cause another singularity if the observation vector $\bar{\rho}$ (a projection of observation vector $\bar{r}$ ) lies anywhere along an edge. In this case, we moved the observation point a very small distance $\left(e_{a}\right.$, it has a range of $\left.10^{-15} \lambda_{0}<e_{b \leq} 10^{-10} \lambda_{0}\right)$ away from the edge, as in [8].

$$
\overline{r_{1}}=\bar{r}_{0}+e_{a} \widehat{u}
$$

where $\bar{r}_{0}$ and $\bar{r}_{1}$ are position vectors of the original and 
updated positions for the observation point and $\hat{u}$ is a unit vector normal to the edge [9].

\section{2-2 Test Setup \& Virtual Vertex}

We examined the angular effect of virtual vertices by first computing the RCS of a $1 \lambda_{0} \times 1 \lambda_{0}$ square conducting plate at $10 \mathrm{MHz}$ at various values of $\theta_{v}$ and $\theta_{r}$. Figs. 2(a) and (b) show the test setup with the inclined conducting plate placed on the infinite ground plane and its equivalent test setup in free-space, respectively. Fig. 2(c) shows a geometrical configuration of the problematic boundary edge, with virtual vertices at various angular positions $\left(\theta_{v}\right)$. We cross-checked the influence of the boundary edges in the half- and free-spaces by attempting to deliberately omit them in each case. We considered four test setups as follows: (1a) no virtual vertices for a structure in Fig. 2(a), (1b) deliberately omitting the boundary edges in Fig. 2(b), (2a) inserting the virtual vertices in Fig. 2(a), and (2b) a structure as in Fig. 2(b) in free-space as a reference.

The starting and ending points of a boundary edge are $v_{1}$ and $v_{2}$, and the center point of the boundary edge is $v_{c}$ (Fig. 1). The virtual vertex associated with the boundary edge is located at a distance $d$ from the center point $v_{c}$. When the angle $\theta_{v}$ is zero, the position of the virtual vertex is $v_{d 0}$ as shown in Fig. 1. The distance $d$ can be represented by the multiplication of a length $L_{e}=\left|v_{1}-v_{2}\right|$ of the boundary edge and a normalized distance $e_{b}=d / L_{e}$ which has a range of $10^{-7}<e_{b} \leq 10^{-1}$ [4]. Using the reference point $v_{d 0}$ and the vertex angle $\theta_{v}$,
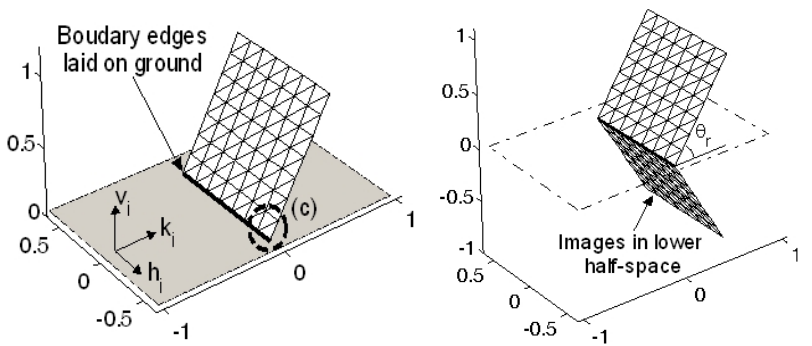

(a) Test setup in half-space (b) Its full-size setup in free-space

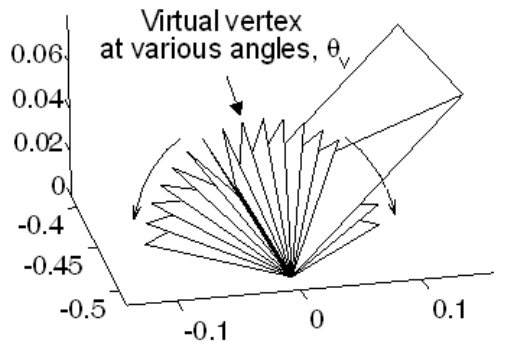

(c) Virtual vertices at various angles $\left(\theta_{v}\right)$

Fig. 2. Test setups with an inclined conducting plate with an inclination angle $\theta_{r}$. a position $v_{d}$ of a virtual vertex placed at a specific angle can be generated as shown in Fig. 2(c).

\section{2-3 Insertion of Virtual Vertices}

The normalized RCS pattern (line with dots) for the test setup (2a) with virtual vertices agrees well with the reference setup (2b), as shown in Fig. 3. However, the normalized RCS pattern (line with diamonds) for the test setup (1a) without virtual vertices does not agree well with the result (line with circles) of the test-setup (2b) for the vv-polarized backscatter, but it agrees with the test setup (1b) that is distorted by deliberately omitting the boundary edge (line with squares).

In all four test conditions, insertion of the virtual vertices affects the RCS at vertical polarization (vvpol.), while horizontal polarization (hh-pol.) is not affected, so that the four lines are placed on top of each other, as shown in Fig. 3. The current sources vertically passing through the boundary edges are relatively agitated by the ground, so the vv-polarized RCS shows significant errors without the addition of the virtual vertices in this calculation.

Fig. 4 shows the polarimetric responses of the test setup (2a) with variation of $\theta_{v}$ from $0^{\circ}$ to $180^{\circ}$. These scanned data along with the angular position of virtual vertex as a function of $\theta_{v}$ can be used to analyze their angular effect by using the reference data, which are the result of the test setup (2b) in free-space. The numbers of interior edges used to solve this scattering problem are 140 and 273 edges, respectively. These scanned data were expressed at $2.5^{\circ}$ intervals and fully computed 5329 times.

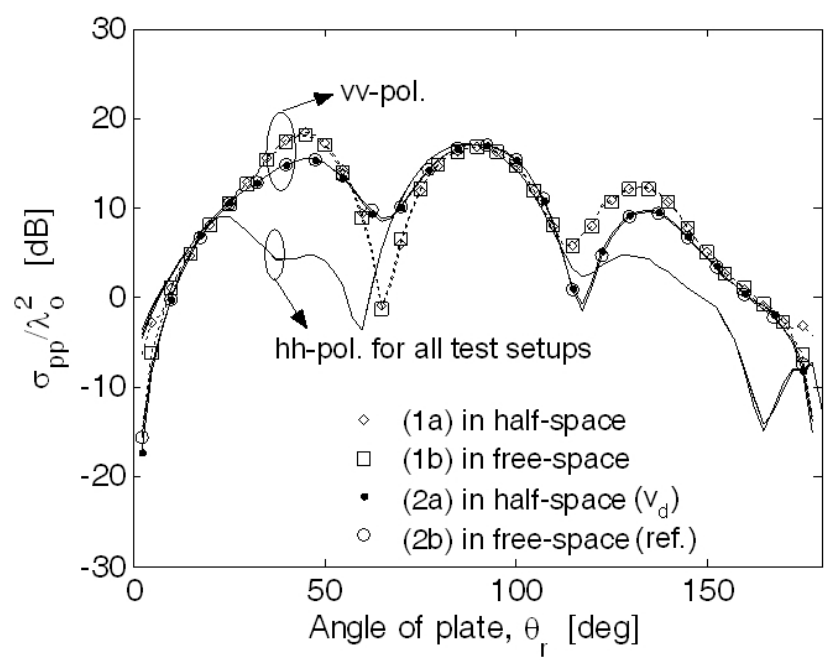

Fig. 3. Scattering property of conducting plates with each test setup: Note that test setup (2a) was computed at reference position, $v_{d 0}$. 


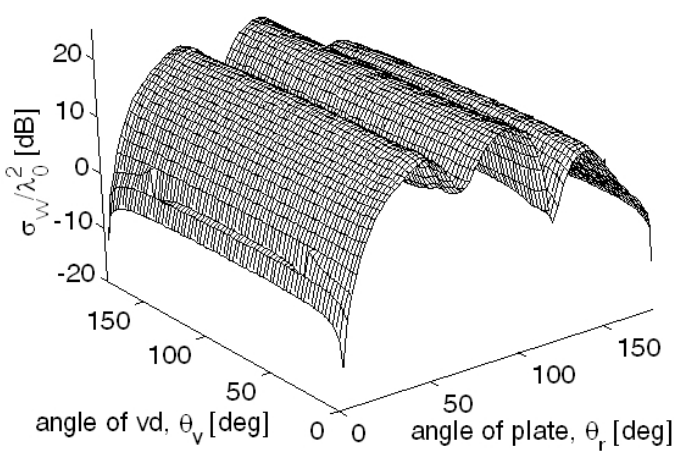

(a) vv-polarization

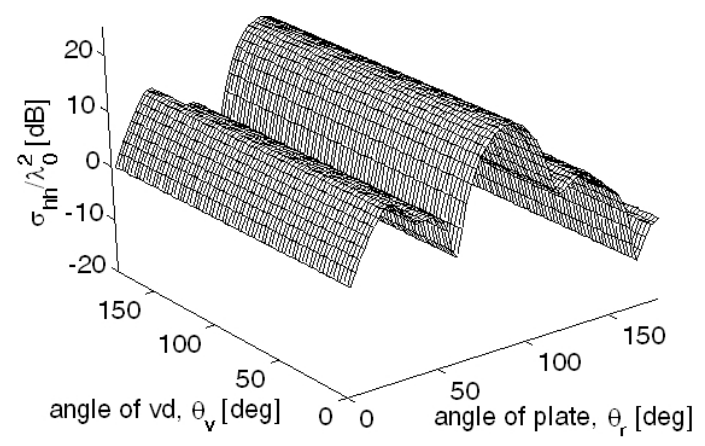

(b) hh-polarization

Fig. 4. Scattering property of a conducting plate at various angles with $0^{\circ} \leq \theta_{v} \leq 180^{\circ}$ and $0^{\circ} \leq \theta_{r} \leq 180^{\circ}$ (using test setup (2a)).

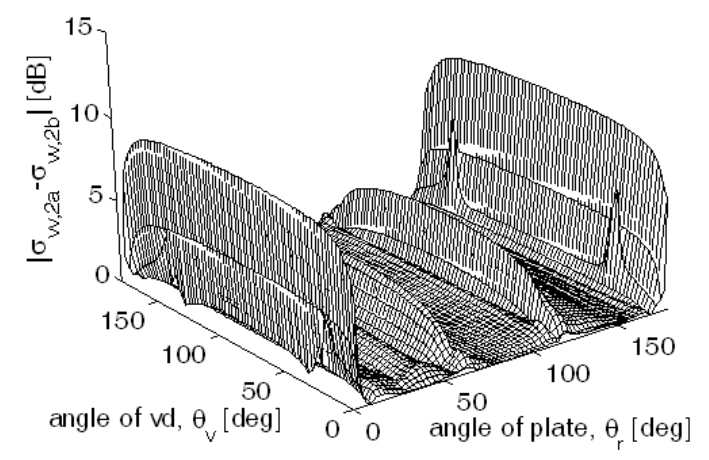

(a) vv-polarization

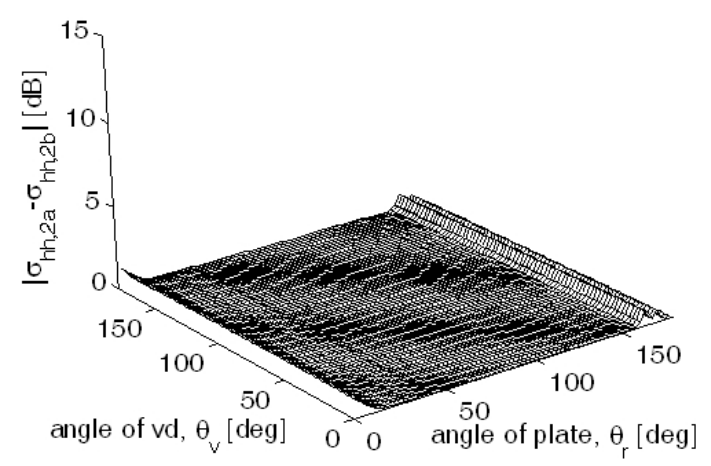

(b) hh-polarization

Fig. 5. Differences between the test setup (2a) with virtual vertices and the test setup ( $2 b)$ as a reference.

\section{Numerical Results}

We analyzed the angular effect of the virtual vertices by computing the differences of the RCSs between the two test setups (2a) and (2b) at various angular positions for vv- and hh-polarizations, as shown in Fig. 5. Both test conditions are equivalent, so the test setup (2b) is considered as a reference to examine the computational accuracy of the test setup (2a), which includes the virtual vertices. In the case of vv-polarization, the difference is maximized when $\theta_{r}$ approaches to $0^{\circ}$ or $180^{\circ}$ because of the inaccuracy at null-points, where the backscatter is seriously insensitive at these regions, such as in the vicinity of $0^{\circ}, 60^{\circ}, 120^{\circ}$ and $180^{\circ}$, as shown in Fig. 5(a).

At near $\theta_{r}=90^{\circ}$, almost no difference exists between the two test setups. The difference is also minimized when the vertex angle $\theta_{v}$ approaches $0^{\circ}$ or $180^{\circ}$, which indicates that the optimum position of the virtual vertex is on the ground plane. On the other hand, at hh-polarization, the difference is lower than $0.05 \mathrm{~dB}$, except at the vicinity of $\theta_{r}=0^{\circ}$ or $180^{\circ}$, as shown in Fig. 5(b).

These data were quantitatively analyzed in more detail by drawing the $\theta_{v^{-}}$and $\theta_{r^{-}}$cuts of Fig. 5(a) for vv-polarization, as shown in Fig. 6(a) and (b), respectively.

Fig. 6(a) illustrates the fact that the virtual vertices at $\theta_{v}=0^{\circ}$ and $180^{\circ}$ have a minimized difference of less than about $0.7 \mathrm{~dB}$ at all inclination angles $\theta_{r}$ of the conducting plate. In particular, it demonstrates optimum performance even at the inclination angle of $\theta_{r}=90^{\circ}$. Therefore, insertion of the virtual vertices is valid even when the surface of a test object placed on a ground plane or their basis vectors are normal to the ground plane. Fig. 6(a) also shows that the difference as a function of $\theta_{v}$ is symmetric regardless of the inclination angle of the test object. In other words, a vertical component of the current source passing through the problematic boundary edge contributes to the MoM process. The synthesized vector to depict them is also sensitive to changes in the angular position of the virtual vertex. Therefore, the magnitude of the vertical component of the synthesized vector is deterministic in this computational process. In addition, Fig. 6(b) shows that the $\theta_{r}$-cut and the difference is also minimized at $\theta_{v}=0^{\circ}$ and $180^{\circ}$, less than about $0.6 \mathrm{~dB}$ in the range of $5^{\circ} \leq \theta_{r} \leq 175^{\circ}$. Two peaks located at $\theta_{r}=65^{\circ}$ and $120^{\circ}$ are related to the inaccurate results at the null-points, as mentioned before. Lastly, a sudden change or singularity caused by an abnormal formation of the virtual vertex is not observed at all angular positions, as shown in Figs. 5 and 6. 


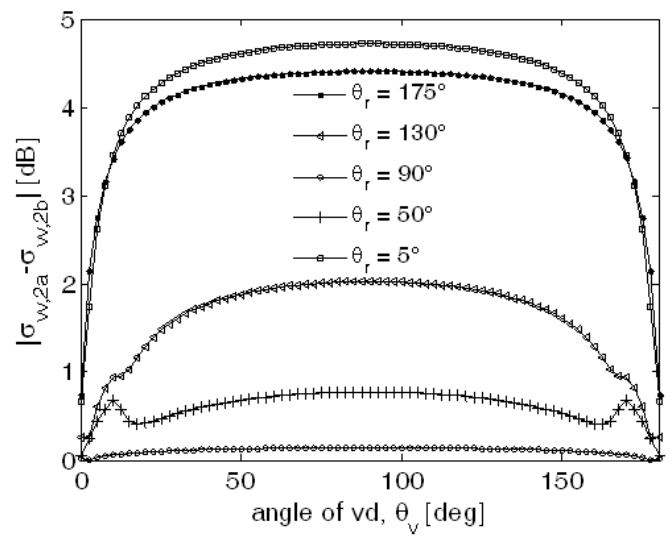

(a) $\theta_{v}$-cut for vv-pol

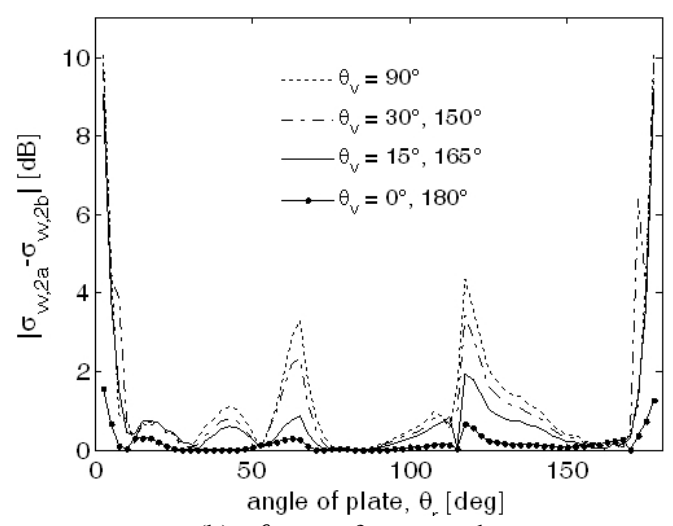

(b) $\theta_{r}$-cut for vv-pol

Fig. 6. Detail views of the angular effect of virtual vertices.

Table 1. Angular effect of the virtual vertex.

\begin{tabular}{|c|c|c|c|}
\hline \multirow{2}{*}{} & \multicolumn{2}{|c|}{ Differences } & \multirow{2}{*}{ Unit } \\
\cline { 2 - 3 } $\begin{array}{c}\text { Optimum } \\
\text { angle of } v_{d} \\
\left(\theta_{v}=0^{\circ}, 180^{\circ}\right)\end{array}$ & $\begin{array}{c}<0.7 \\
(\text { max. error } \\
\left.\text { at } \theta_{r}=5^{\circ}\right)\end{array}$ & $<0.05$ & holarization \\
\hline \multirow{2}{*}{ Note } & 1) Optimum angles of $v_{d}\left(\theta_{v}=0^{\circ}, 180^{\circ}\right)$ are \\
& $\begin{array}{l}\text { valid in all test conditions. } \\
\text { 2) Arbitrarily positioned } v_{d} \text { nearly not affects } \\
\text { hh-polarization. }\end{array}$ \\
\hline
\end{tabular}

In addition, this proposed method can be compared with the existing method for the half-space scattering problem. This performance is also summarized as shown in Table 2. In particular, when a metal hemisphere on an infinite ground plane was used as a test target, it consists of two kinds of hemispheres, with and without a bottom side in contact with the interface of the halfspace [4]. Extraction of the singularity caused by the bottom side cells also requires an additional sub-function in the MoM process. This may affect the delay, thereby increasing the processing time and poor accuracy.
Table 2. Comparison results of the proposed virtual vertex technique using a metal hemisphere on ground surface.

\begin{tabular}{|c|c|c|c|}
\hline & \multicolumn{2}{|c|}{ MoM process } & \multirow{2}{*}{ Unit } \\
\hline & Existing & Proposed, $\left(v_{d}\right)$ & \\
\hline $\begin{array}{l}\text { Impedance } \\
\text { matrix, [Z] }\end{array}$ & $\begin{array}{l}\text { (a) } 1000 \times 1000 \text {, } \\
\text { (b) } 500 \times 500\end{array}$ & $536 \times 536$ & [edges] \\
\hline $\begin{array}{l}\text { Processing } \\
\text { time }\end{array}$ & $7.5,1.5$ & 2.02 & {$[\mathrm{~min}]$} \\
\hline $\begin{array}{l}\text { Accuracy } \\
\text { (vv-pol.) }\end{array}$ & $-4.7,-4.5$ & $<0.5$ & {$[\mathrm{~dB}]$} \\
\hline $\begin{array}{l}\text { Test con- } \\
\text { ditions }\end{array}$ & \multicolumn{3}{|c|}{$\begin{array}{l}\text { 1) Target: metal hemisphere }\left(k_{0} a=0.5\right) \text { on an } \\
\text { infinite ground plane } \\
\text { 2) Accuracy: Mie-series solution [11] for me } \\
\text { tal sphere vs numerical results. ( }{ }^{(a)} \text { closed- } \\
\&{ }^{(b)} \text { open-bottom hemisphere) [4] } \\
\text { 3) Computation: CPU } 3.4 \mathrm{GHz} \text {, RAM } 2 \mathrm{~GB}\end{array}$} \\
\hline
\end{tabular}

\section{Conclusion}

We investigated the angular effect of the virtual vertices inserted to treat the boundary edges on an infinite conducting surface in this study. A vertical component of the synthesized vector that expresses the current source passing through the problematic boundary edge was identified as contributing to the MoM process for halfspace scattering. Therefore, the angular position of a virtual vertex should be selected to be maximized as a vertical component of the synthesized vector. The optimum angular position of the virtual vertices might lie on the ground plane, i.e., $\theta_{v}=0^{\circ}$ or $180^{\circ}$, regardless of the inclination angle of the triangle cell associated with the problematic boundary edge. This virtual vertex technique also improves the accuracy of the RCS computation of a half-space scattering problem for all cases, regardless of the angle of $\theta_{r}$, even when the angle between $\theta_{v}$ and $\theta_{r}$ is $90^{\circ}$. In addition, the technique effectively manages the number of interior edges by the reduction of physical redundancy of triangular cells on ground surface.

Finally, this proposed technique will contribute to increased accuracy of the numerical model for analyzing the scattering property of scattering particles above the ground surface in the field of microwave remote sensing.

Authors thank the anonymous reviewers for their thoughtful comments. This work was supported by Global Surveillance Research Center (GSRC) program funded by the Defense Acquisition Program Administration (DAPA) and Agency for Defense Development (ADD). 


\section{References}

[1] S. M. Rao, D. R. Wilton, and A. W. Glisson, "Electromagnetic scattering by surfaces of arbitrary shape," IEEE Trans. Antennas Propag., vol. AP-30, no. 3, pp. 409-418, May 1982.

[2] Pasi Yla-Oijala, Matti Taskinen, "Calculation of CFIE impedance matrix elements with RWG and $\mathrm{n} \times$ RWG functions," IEEE Trans. Antennas Propag., vol. 51, no. 8, pp. 1837-1846, Aug. 2003.

[3] Y. Q. Hu, J. J. Ding, D. Z. Ding, and R. S. Chen, "Analysis of electromagnetic scattering from dielectric objects above a lossy half-space by multiresolution preconditioned multilevel fast multipole algorithm," IET Microw. Antennas Propag., vol. 4, Iss. 2, pp. 232239, 2010.

[4] J. -H. Hwang, Y. Oh, "Investigation of the effect of boundary edges placed on an infinite conducting surface and effective treatment using virtual vertices," IEEE Antennas and Wireless Propagation Letters, vol. 11, pp. 913-916, Aug. 2012.

[5] Y. Oh, Y. M. Jang, and K. Sarabandi, "Full-wave analysis of microwave scattering from short vegetation: an investigation on the effect of multiple scattering," IEEE Trans. Geosci. Remote Sensing, vol. 40, no.

\section{Ji-Hwan Hwang}

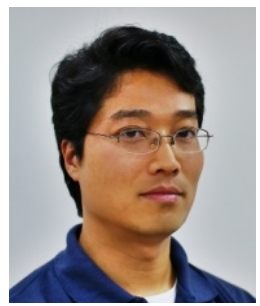

received the B.S. degree in radio engineering and the M.S. degree in radio science and communication engineering from Hongik University, Seoul, Korea, in 2001 and 2003, respectively. He worked at LG Electronics Co. from 2003 to 2006. $\mathrm{He}$ is currently a Ph.D. candidate in the Department of Electronic Information and Communication Engineering, Hongik University, Seoul, Korea. His research interests include microwave scattering, microwave remote sensing, and ground-based SAR.

\section{Soon-Koo Kweon}

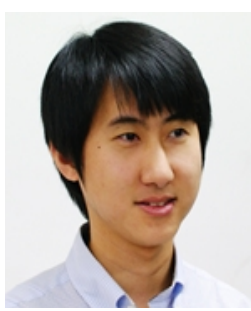

received the B.S. degree in electronic and electrical engineering and the M.S. degree in electronic information and communication engineering from Hongik University, Seoul, Korea, in 2007 and 2009, respectively. He is currently a Ph.D. candidate in the Department of Electronic Information and Communication Engineering, Hongik University, Seoul, Korea. His research interests include microwave scattering, microwave remote sensing, and SAR image analysis.
11, pp. 2522-2526, Nov. 2002.

[6] I. V. Lindell, E. Alanen, "Exact image theory for the sommerfield half-space problem, part I: vertical magnetic dipole," IEEE Trans. Antennas Propag., vol. AP-32, no. 2, pp. 126-133, Feb. 1984.

[7] I. V. Lindell, E. Alanen, "Exact image theory for the sommerfield half-space problem, part II: Vertical electric dipole," IEEE Trans. Antennas Propag., vol. AP-32, no. 8, pp. 841-847, Aug. 1984.

[8] W. C. Gibson, The Method of Moments in Electromagnetics, Chapman \& Hall/CRC, Boca Raton, FL, pp. 161-270, 2008.

[9] D. R. Wilton, S. M. Rao, A. W. Glisson, D. H. Schaubert, O. M. Al-bundak, and C. M. Butler, "Potential integrals for uniform and linear source distributions on polygonal and polyhedral domain," IEEE Trans. Antennas Propag., vol. AP-32, no. 3, pp. 276281, May 1984.

[10] C. T. Tai, Dyadic Green Functions in Electromagnetic Theory, IEEE Press, NJ, $2^{\text {nd }}$ Edition, pp. 9295, 1994.

[11] G. T. Ruck, D. E. Barrick, W. D. Stuart, and C. K. Krichbaum, Radar Cross Section Handbook, New York: Plenum, pp. 141-159, 1970.

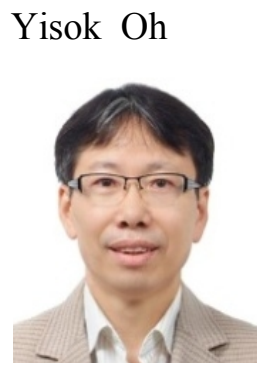

received the B.S. degree in electrical engineering from Yonsei University, Seoul, Korea, in 1982, the M.S. degree in electrical engineering from the University of Missouri-Rolla, in 1988, and the Ph.D. degree in electrical engineering from the University of Michigan, Ann Arbor, in 1993. Since 1994, he has been with the School of Electronic and Electrical Engineering, Hongik University, Seoul, Korea. Currently, he is a Professor at Hongik University, and an IEEE Fellow. His current research interests include microwave remote sensing, antennas, and radio wave propagation. 